\title{
Theoretical Analysis of Formation and Sustainment Methods for Compact Toroids
}

\author{
Ricardo Farengo, Agustín F. Lifschitz, Hugo E. Ferrari, Sebastián Bouzat, \\ Centro Atómico Bariloche e Instituto Balseiro, 8400 S. C. de Bariloche, RN, Argentina \\ and Roberto A. Clemente \\ Instituto de Física Gleb Wataghin, Universidade Estadual de Campinas, 13083-970 Campinas, Brazil
}

Received on 19 January, 2004; revised version received 21 April, 2004

\begin{abstract}
Recent theoretical studies on the use of neutral beams (NB), rotating magnetic fields (RMF) and helicity injection (HI) to form and sustain compact toroids are reported. A Monte Carlo code was employed to study NB injection in Field Reversed Configurations (FRC) and Spheromaks. The code calculates the ionization of the neutral particles and follows the exact orbits of the ions. The magnetic field and density profiles are determined by solving a Grad-Shafranov equation that includes the beam current. RMF current drive in FRCs was studied using a fully 2D code that solves the two fluid equations with massless electrons and uniform temperature. The ion momentum equation includes viscosity and collisions with electrons and neutrals. The electrons are described using an Ohm's law with the Hall and pressure gradient terms. Ion spin up due to collisions with electrons reduces the current drive efficiency and a large fraction of neutrals is needed to keep the azimuthal ion velocity small. The principle of minimum rate of energy dissipation was employed to calculate relaxed states for a flux core spheromak sustained by helicity injection. States with large regions of closed flux surfaces and significant toroidal current were found. Changing the resistivity profile modifies the safety factor profile, which can change from one that has a maximum at the magnetic axis (for uniform resistivity) to a tokamak-like $q$-profile.
\end{abstract}

\section{Introduction}

Although the tokamak is the most advanced magnetic confinement concept, a commercial power plant utilizing a standard tokamak would be large and expensive and have a relatively high electricity cost. The recognition of this problem has prompted, in recent years, a renewed interest in concepts that have lower development costs and could result in smaller, less expensive, reactors. A family of toroidal devices, generically known as Compact Toroids (CT), could satisfy these requirements.

Compact toroids are defined as toroidal configurations that have no structural elements linking the torus. Their aspect ratio is 1 and offer the possibility of translating the configuration after formation. Compact toroids can operate with high values of $\beta$ and are ideally suited to burn advanced fuels that produce fewer, or no neutrons. The different configurations are classified according to the ratio between the toroidal and poloidal fields. The most advanced CTs are the Field Reversed Configuration (FRC) [1] and the Speromak [2]. FRCs are elongated, have negligible toroidal field and operate with $\beta$ close to 1 . Spheromaks have toroidal and poloidal fields of the same order, produced by plasma currents, and lower values of $\beta$.

The development of efficient formation and sustainment methods is a key issue for all magnetic confinement concepts, in particular CTs. In standard tokamaks, start-up and long pulse operation can be achieved using only the ohmic heating $(\mathrm{OH})$ coil but the topology of CTs prevents the use of a central solenoid.

The traditional method used to form FRCs, field reversed $\theta$-pinch, requires high voltages and does not appear scalable to reactor-size configurations. In addition, this method can not be used to sustain the configuration after formation. The most promising method to form and sustain FRCs is rotating magnetic field (RMF) current drive. This method has been used to produce cold rotamak configurations [3] and is currently under investigation in larger, more energetic, devices at the University of Washington [4]. Neutral beams (NB) could be used to form an FRC, to sustain the current and/or to heat the plasma. In addition, the use of beams to improve the stability of FRCs has been also proposed. The ARTEMIS [5] reactor design involves the use of beams and initial experiments with a $10 \mathrm{keV}, 10 \mathrm{~A}$ neutral $\mathrm{H}$ beam have been performed [6].

Although various methods for spheromak formation and sustainment have been demonstrated, helicity injection (HI) has been the most successful [2]. DC HI is currently employed in the SSPX spheromak [7] and AC HI is employed in HIT-SI [8]. Since HI current drive relies on relaxation, the calculation of relaxed states consistent with the boundary conditions needed for HI is clearly of interest.

In this paper we present the results of theoretical analysis regarding the use of NBI, RMF and HI to form and sus- 
tain FRCs and Spheromaks. The structure of the paper is as follows. In section 2 we consider NBI in FRCs and Spheromaks; in section 3 the use of RMFs to sustain the current in FRCs is analyzed and in section 4 relaxed states for a flux core spheromak sustained by helicity injection are presented. Finally, in section 5, we summarize and discuss our results.

\section{Neutral Beam Injection in FRCs and Spheromaks}

Neutral beam injection is one of the methods proposed to sustain the current in FRCs. It could be used alone or combined with RMF to cancel the torque transferred by the RMF driven electrons to the ions. Neutral beams have been injected in the FIX device [6] but, do to the low magnetic field, the beam was directed primarily in the axial direction and confined by strong end mirrors. In addition, the lifetime of the experiments was much shorter than the slowing down and thermalization times, thus limiting the driven current and the deposited power. Spheromaks are generally formed and sustained by helicity injection and alternative methods are usually not considered. However, using NBI to drive part of the current could reduce the amplitude of the fluctuations needed to sustain the configuration, thus improving the confinement, and provide additional control over the current profile. Finally, injecting NBs in FRCs and Spheromaks will heat the plasma and provide a population of energetic particles that could improve the stability of both configurations.

To study NBI current drive and heating in compact toroids a Monte Carlo code was developed. Assuming a continuous injection of neutral particles the code calculates their ionization and follows the exact trajectories of the ions. This information is used to reconstruct the spatial distribution of the beam density, current and transferred power and momentum in steady state. The effect of Coulomb collisions is introduced via a Fokker-Planck collision operator. The magnetic field and density profiles needed to calculate the orbits and the stopping are determined by solving a GradShafranov equation that contains the contribution of the beam to the total current but not to the pressure. The equilibrium and the beam current are calculated iteratively, keeping constant the average plasma density, and the poloidal plasma current in the case of Spheromaks, until the solution converges. The relevant beam variables are the axial position (where the beam is injected), the injection angle $(\alpha)$, the neutral injection current $\left(I_{N}\right)$, the energy of the beam particles $\left(E_{N}\right)$ and the impact parameter $(b)$. Fig. 1 presents a schematic diagram showing the injection geometry.
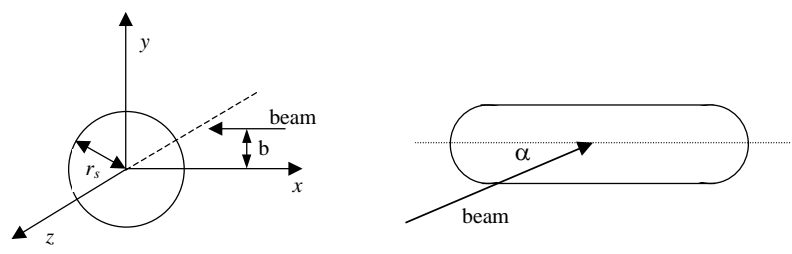

Figure 1. Injection geometry for FRCs.

\section{$2.1 \quad$ FRCs}

Studies for conditions similar to the best ones achieved in experimental devices [9] show the feasibility of using NBI for current drive in FRC. Fig. 2 shows the spatial distribution of beam current and the flux surfaces for a low current beam $\left(I_{N}=1 \mathrm{~A}\right)$ and a high current one $\left(I_{N}=300 \mathrm{~A}\right)$ injected in an equlibrium with a racetrack-like separatrix and a hollow current profile. The FRC parameters are: separatrix radius $r_{s}=30 \mathrm{~cm}$, lenght $z_{s}=240 \mathrm{~cm}$, external field $B_{\text {ext }}=5 \mathrm{kG}$ and temperatures $T_{e}=T_{i}=0.5 \mathrm{keV}$. The beam is injected at the midplane, perpendicular to the FRC axis and has an energy $\left(E_{N}\right)$ of $20 \mathrm{keV}$. In the low current case the beam spreads over all the length of the FRC due to diffusion produced by collisions with the plasma ions. The beam current has a double peaked radial profile due to the radial oscillation of the particles around the magnetic null. In the high current case there is a significant change in the FRC equilibrium. The beam current increases the magnetic field near the injection region, producing a self-confining effect which reduces the axial excursion of the deflected beam ions. Due to the high $\beta$, the increase in the magnetic field around the injection region produces a similar increase in the density. Since the stopping increases with the density, the beam current increases slower than linear with $I_{N}$.
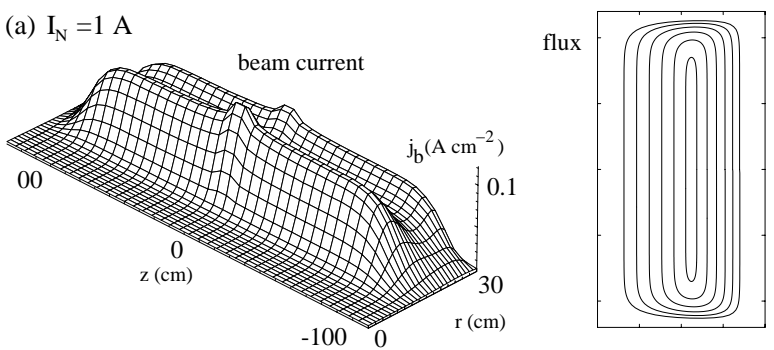

(b) $\mathrm{I}_{\mathrm{N}}=300 \mathrm{~A}$
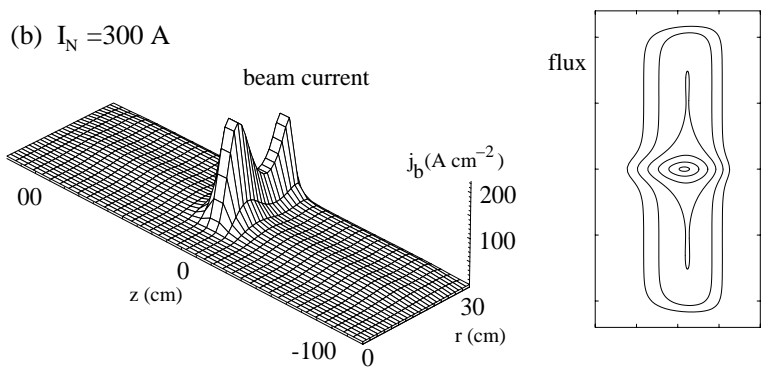

Figure 2. Spatial distribution of beam current and flux surfaces for a $20 \mathrm{keV}$ beam. (a): $I_{N}=1 \mathrm{~A},(\mathrm{~b}): I_{N}=100 \mathrm{~A}$. 

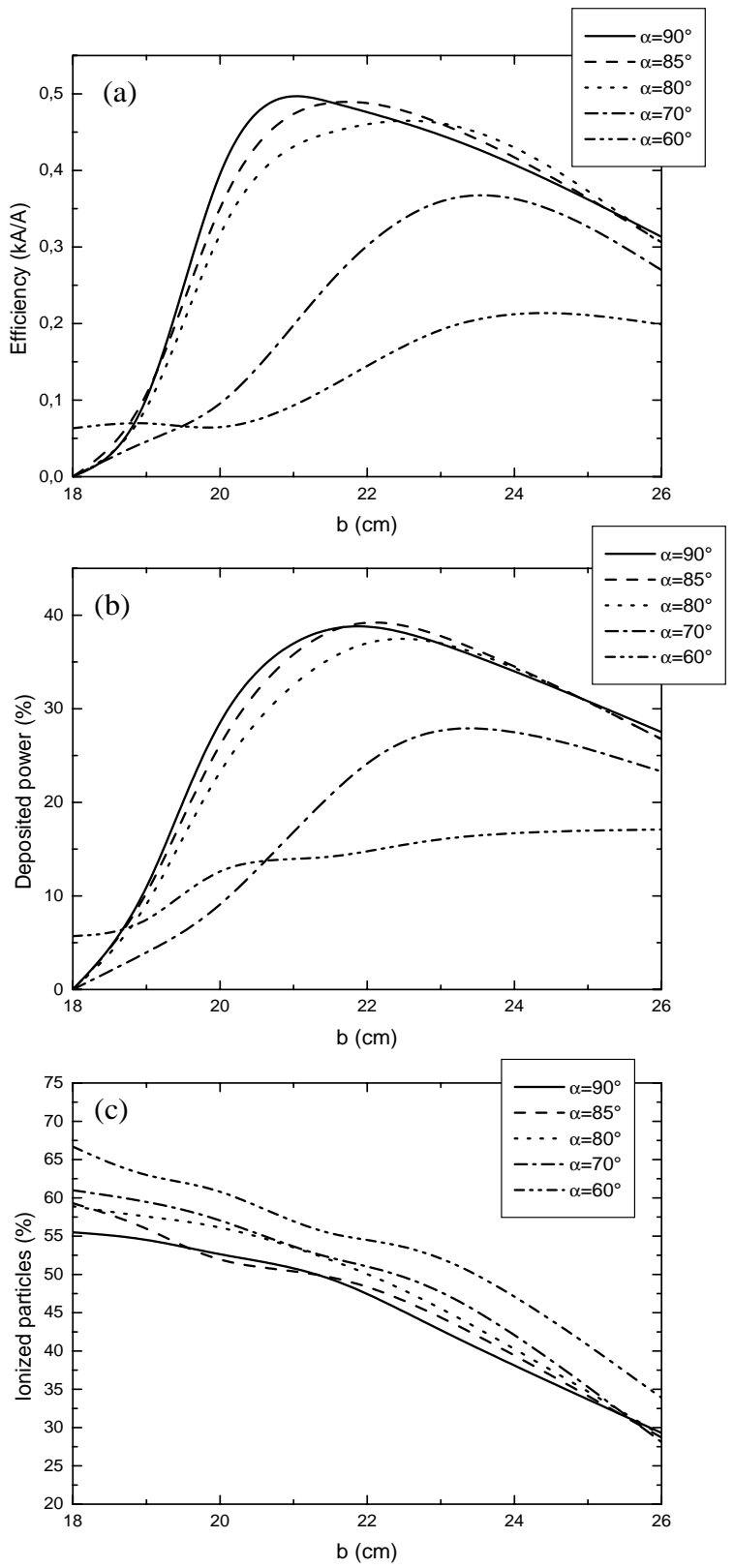

Figure 3. Current drive efficiency (3.a), deposited power (3.b) and fraction of ionized particles (3.c) as a function of the impact parameter (b) for selected values of $\alpha$.

In recent studies, NBI into the TCS device [4] was considered. In TCS, FRCs are formed and sustained using RMF and have lower magnetic fields (than in the studies reported above $)$ and higher $\chi_{s}\left(\chi_{s}=r_{s} / r_{w} \approx 0.8-0.9, r_{w}\right.$ wall radius). These two facts restrict the allowable range of energies and the injection geometries. Our calculations show that for energies of $10 \mathrm{keV}$ or more Deuterium beams can not be trapped. Hydrogen beams were therefore employed in the calculations. Another factor affecting beam confinement is the presence of the transverse rotating magnetic field that allows particles to move radially and collide with the wall. We show here the results for the best conditions predicted for TCS, i.e. external field $B_{\text {ext }}=750 \mathrm{G}$,
$T=140 \mathrm{eV}$, peak plasma density $n_{\text {peak }}=0.5 \times 10^{14} \mathrm{~cm}^{-3}$, and $\chi_{s}=0.8$. The loss of particles through the ends is prevented by including magnetic mirrors, with a mirror ratio of 2.7. This value is large enough to reflect almost all the particles that reach the mirror region. Beams injected through the ends and almost tangentially (angle with respect to the FRC axis $\alpha \approx 30^{\circ}$ ) do not drive a significant current and deposit less that $5 \%$ of their power into the plasma, due to loss of particles by collisions with the wall. In the cases presented below the beams are injected at the midplane with an energy of $10 \mathrm{keV}$.

Figure 3 shows the current drive efficiency (3.a), the deposited power (3.b) and the fraction of ionized particles (3.c) as a function of the impact parameter $b$ for selected values of $\alpha$. For perpendicular injection $\left(\alpha=90^{\circ}\right)$ the efficiency and the deposited power present a maximum around $21 \mathrm{~cm}$, close to the null radius $r_{0} \approx 20.5 \mathrm{~cm}$. As the angle is reduced, the maximum shifts toward larger values of the impact parameter. For $\alpha$ between $80^{\circ}$ and $90^{\circ}$ the maximum values of the efficiency and the deposited power remain approximately constant at $0.5 \mathrm{kA} / \mathrm{A}$ and $42 \%$ respectively. Particles injected with small $b$ are trapped in orbits that get very close to the wall and collide with it after few collisions, or even hit the wall before being deflected at all. This loss of particles causes a reduction of the efficiency for $b<18 \mathrm{~cm}$ in spite of the fact that for these values of $b$ the ionization fraction is the largest (Fig. 3.c). When $b$ is larger than $22-23 \mathrm{~cm}$, the fraction of ionized particles decreases rapidly due to the lower plasma density encountered by the neutral beam particles. This results in a reduction of the current drive efficiency and the deposited power.

To explore the effect of the RMF, we included in the simulation a transverse rotating field. Approximate analytical expressions, taken from Ref. [10] were employed for the radial dependence of the RMF field, no axial dependence was included. The RMF is determined by three parameters, the field magnitude outside the separatrix $B_{\omega}$, the penetration range $\delta$ and the rotation frequency $\omega$. Fig. 4 shows the efficiency as a function of $B_{\omega}$ (4.a), $\delta$ (4.b) and $\omega$ (4.c). The axial FRC field is $750 \mathrm{G}$. For a rotation frequency of $1 \mathrm{Mrad} / \mathrm{s}$ and a small penetration range $\delta=0.1 r_{s}$ (fig. 4.a), the efficiency decreases rapidly for $B_{\omega}>40 \mathrm{G}$, dropping to less than $10 \%$ of the value without RMF for $B_{\omega}=100 \mathrm{G}$. The efficiency also decreases with the penetration range $\delta$ (4.b). The dependence with the rotation frequency is more complex (4.c). For $\omega=0\left(B_{\omega}=50 \mathrm{G}\right.$ and $\left.\delta=0.1 r_{s}\right)$, the efficiency is roughly $60 \%$ of the value without RMF and decreases sharply for $\omega \approx 5 \times 10^{6} \mathrm{~s}^{-1}$. This is due to a resonance between the frequency of the RMF and the frequency of rotation of the beam ions around the FRC axis. At this frequency, the RMF is almost static in the beam reference frame, enhancing the transversal displacement of beam particles and therefore the losses by collisions with the wall. For higher frequencies the efficiency grows again and a second broad resonance appears around $12 \mathrm{Mrad} / \mathrm{s}$. TCS typically operates with frequencies between 0.5 and $1 \mathrm{MHz}$, far from the resonances. 
(a)

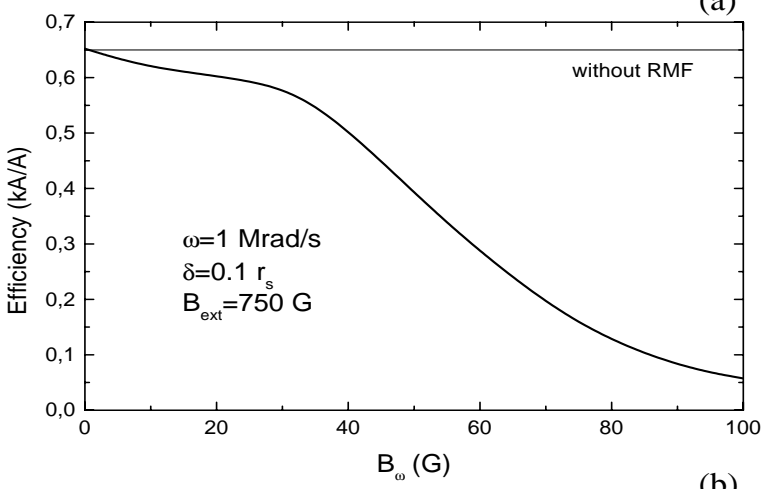

(b)

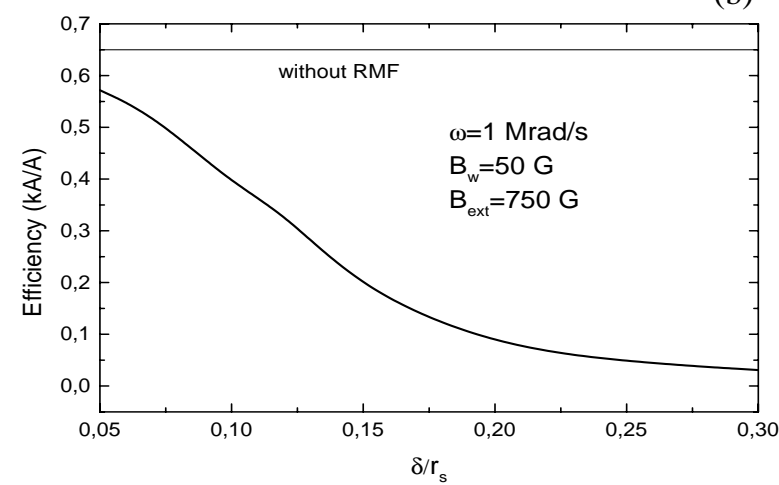

(c)

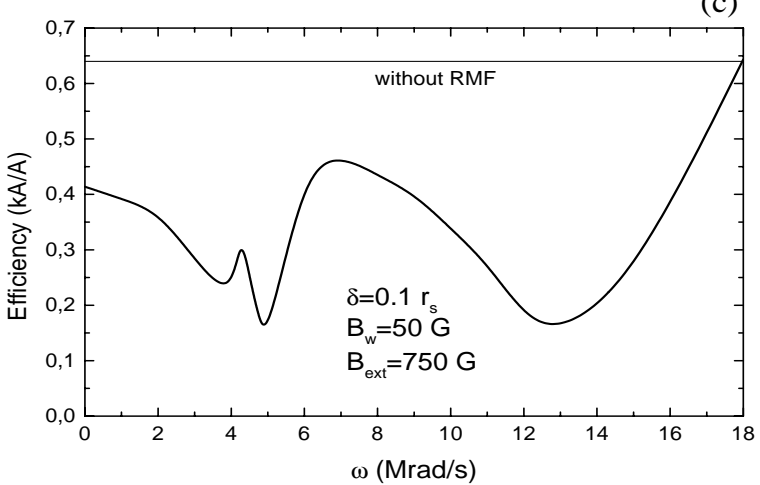

Figure 4. Efficiency as a function of $B_{\omega}$ (4.a), $\delta$ (4.b) and $\omega$ (4.c).

\subsection{Spheromaks}

The plasma is considered to be inside a flux conserver of radius $R_{s}$ and height $Z_{s}$, and the polodal flux is taken to be zero at the boundary. The injection geometry is shown in Fig. 5. The results presented here were obtained with $r_{s}=z_{s}=50 \mathrm{~cm}, r_{0}=31 \mathrm{~cm}, n=10^{14} \mathrm{~cm}^{-3}$, $T_{e}=T_{i}=0.5 \mathrm{keV}, B_{\text {ext }}=7 \mathrm{kG}$ and a peaked density profile [11]. The current driven by the beam is the beam current minus the electron cancellation current, which is due to the drag of plasma electrons by the beam. The maximum cancellation occurs at the magnetic axis, where there are no electrons trapped in banana-like orbits. Fig. 6 shows the total driven current per unit of injected power. The curves for $Z_{\text {eff }}=1$ and $Z_{\text {eff }}=1.86$ are displayed. The current presents a broad maximum around $40 \mathrm{keV}$. The beam current is higher for $Z_{\text {eff }}=1$ than for $Z_{\text {eff }}=1.86$, but the total driven current is lower in the former case. The reduction of the plasma effective charge does not result in an improvement in the total current drive efficiency because the reduction of the stopping cross section is compensated by an increase in the electron cancellation current.

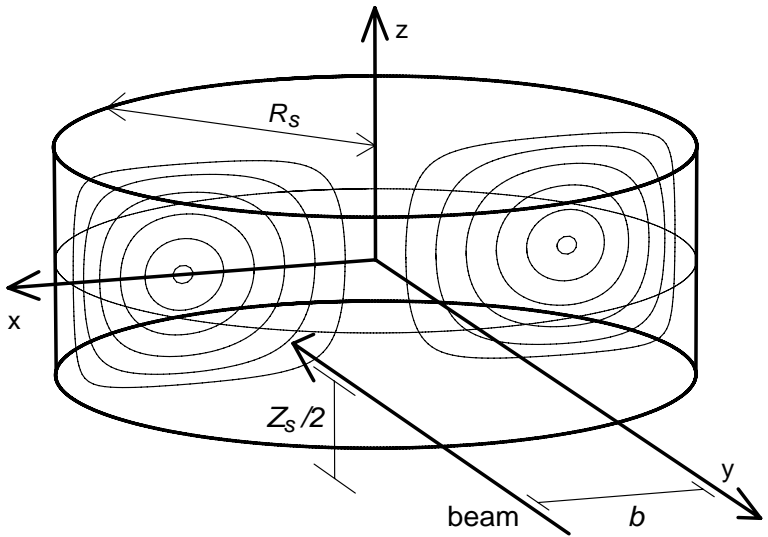

Figure 5. Injection geometry for spheromaks.

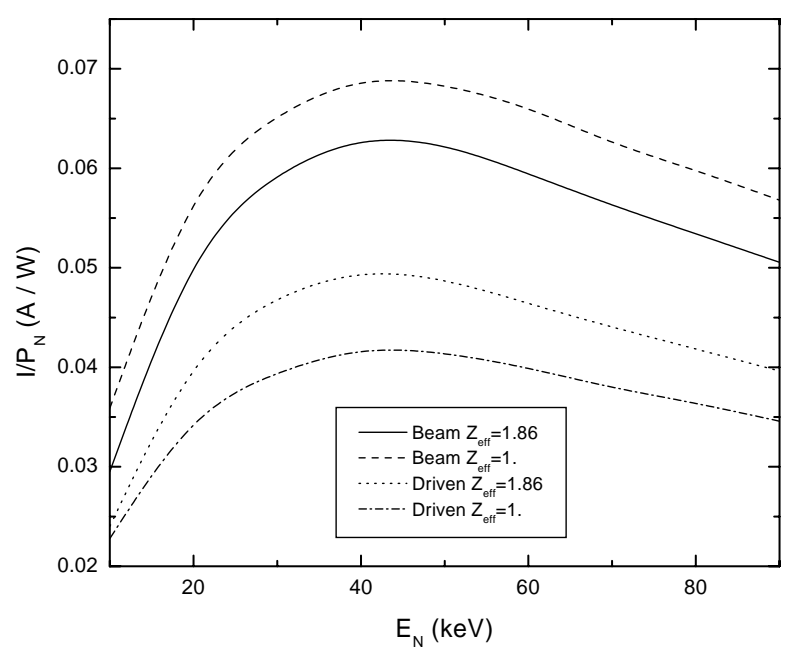

Figure 6. Total driven current per unit of injected power as a function of $E_{N}$.

The beam current profiles are broad for injection with $b<r_{0}$, concentrated around the magnetic axis for $b=r_{0}$ and hollow for $b>r_{0}$ (Fig. 7). The electron cancellation current is larger for $b=r_{0}$ because the beam concentrates in the region with the smallest fraction of trapped electrons. For low $Z_{e f f}$, the cancellation current is almost half the beam current. The safety factor profiles of the selfconsistent equilibria show a clear sensitivity to the impact parameter (Fig. 8). When $b=r_{0}$, the current concentrates around the axis thus reducing $q_{0}$. When $b>r_{0}$, the hollow current profiles increases $q_{0}$. Finally, when $b<r_{0}$ there is a small reduction in $q$. The power deposition distribution can 
be controlled even with the simple injection geometries employed here, where only $b$ is allowed to change. For injection below the magnetic axis $\left(b<r_{0}\right)$, the power is deposited in a broad region that becomes more concentrated around the axis as $b$ approaches $r_{0}$. For $b>r_{0}$ the profile is hollow and the power deposited in the plasma core is small.
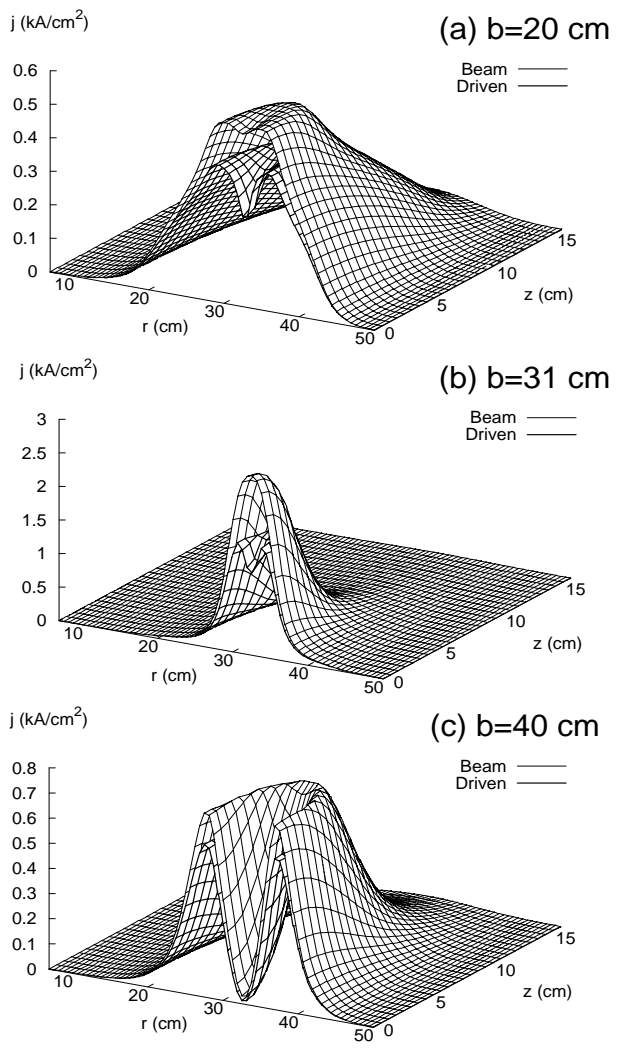

Figure 7. Spatial distribution of the current density for different values of the impact parameter.

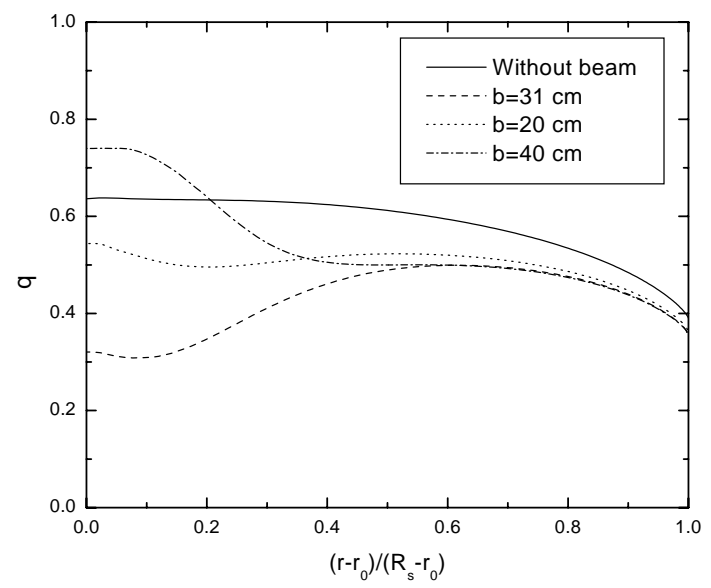

Figure 8. Safety factor profiles for different values of the impact parameter.

\section{Rotating Magnetic Field Current Drive}

Extensive theoretical studies of RMF current drive in FRCs have been performed in the last 20 years [12]-[15]. However, none of these studies has included the full ion dynamics in a time dependent calculation. Although ideally the RMF drags only the electrons, it is clear that electron ion collisions will accelerate the ions in the azimuthal direction, thus reducing the total current. It is therefore important to study ion acceleration, its effect on the equilibrium, and the mechanisms that can prevent it. We studied the effect of ion motion on RMF current drive in FRCs using a 2D $(r, \theta)$ numerical code that solves a set of equations for $A_{z}, B_{z}$, the three components of the ion velocity and the density $\left(n_{e}=n_{i}\right)$. Viscosity and collisions with neutrals, which remain fixed, were included in the ion momentum equation.

In fixed ion calculations there are only two dimensionless parameters: $\lambda=r_{s}\left(\mu_{0} \omega / 2 \eta\right)^{1 / 2}$ and $\gamma=B_{\omega} / e n \eta$, where $\eta$ is the resistivity. When the full ion dynamics is included new dimensionless parameters appear in the equations. They are: $\delta=2 \operatorname{Ten}_{0} \mu_{0} / m_{i} \omega B_{\omega}, \Omega=e B_{\omega} / m_{i} \omega$ and $B_{0}=B_{\text {ext }} / B_{\omega}$. The boundary conditions imposed at the plasma vacuum interface are: continuity of $\partial A_{z} / \partial r$ and $\partial v_{i \theta} / \partial r, v_{i r}=0, \partial v_{i z} / \partial r=0$ and $\partial n / \partial r=0$. In the calculation of the efficiency the maximum current is defined with the ions fixed.

For values of $\gamma$ and $\lambda$ which result in full penetration of the RMF and synchronous electron rotation the ions accelerate in the azimuthal direction and their final velocity depends on the fraction of neutrals $\left(f_{n}=n / n_{\text {neutrals }}\right)$. For the parameters considered $(\gamma=20, \lambda=11, \delta=2.9, \Omega=$ 0.2 and $\left.B_{0}=3\right), v_{i \theta} / v_{r m f}=0.08$ and 0.45 for $f_{n}=50 \%$ and $10 \%$ respectively $\left(v_{r m f}\right.$ : velocity for synchronous rotation). Ion rotation reduces the efficiency (current). This is shown in Fig. 9, which presents a plot of the efficiency as a function of normalized time $(\tau=\omega t)$ for different fractions of neutral atoms and also for fixed ions. The dimensionless parameters are the same as above. There are two stages. In the first stage the efficiency grows and in the second one it decays $\left(f_{n}<10 \%\right)$ or remains approximately constant. The fastest initial growth is obtained when the are no neutrals and the slowest one when the ions remain fixed. The faster initial growth obtained with the smaller values of $f_{n}$ is the result of two effects. First, the ions rotate as a rigid rotor across the entire cross section due to their viscosity. This means that the ions rotate in regions where the RMF has not yet penetrated. Second, the centrifugal force pushes the ions outwards reducing the density inside the plasma and increasing the effective $\gamma$ seen by the RMF. Since the highest $v_{i \theta}$ is obtained when $f_{n}=0 \%$, the effective $\gamma$ and the RMF penetration rate are the highest in this case. Once the RMF has completely penetrated into the plasma, ion rotation reduces the total plasma current. 


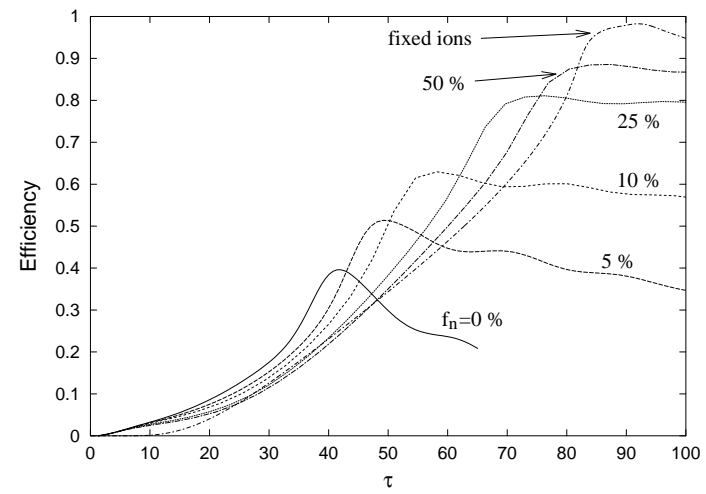

Figure 9. Efficiency as a function of time for different values of the fraction of neutrals and for fixed ions. $\gamma=20, \lambda=11, \delta=2.9, \Omega=0.2$ $B_{0}=3$.

When $\gamma$ is below the value corresponding to full penetration, the efficiency can be higher for moving ions and variable density than for fixed ions. Fig. 10 shows the time evolution of the efficiency for $\gamma=14, \lambda=11$ and the other parameters as in Fig. 9. The efficiency obtained including ion motion, and $f_{n}=50 \%, 25 \%$ and $10 \%$, is higher than the efficiency of the fixed ion model. This increase in the efficiency is due to a growth in the penetration range which is not overcomed by ion rotation when $f_{n}>10 \%$. As the RMF penetrates, the ions begin to rotate everywhere and centrifugal effects reduce the density at the center of the plasma column. The density reduction increases the local value of $\gamma$ actually seen by the RMF above the critical value needed for penetration. This process allows the system to access steady states with large penetration and high density at the edge that, a priori, could have been considered unaccessibles.

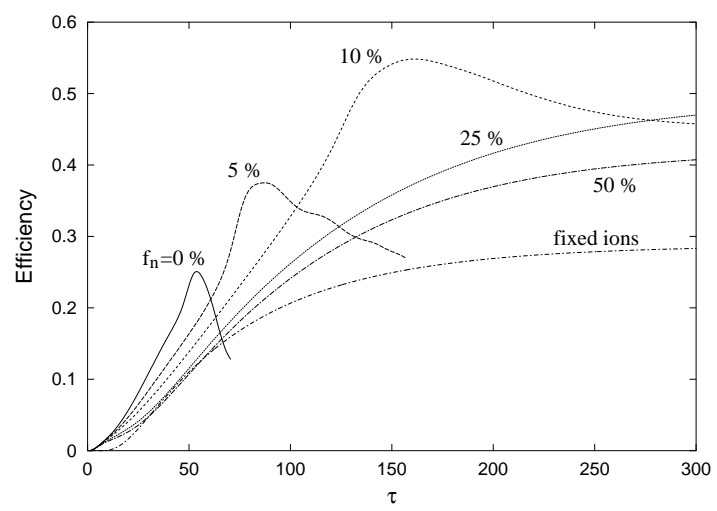

Figure 10. Efficiency as a function of time for different values of the fraction of neutrals and for fixed ions. $\gamma=14, \lambda=11, \delta=2.9$, $\Omega=0.2 \quad B_{0}=3$.

When the ion rotation is large enough and the radial density profile has a maximum inside the plasma an azimuthal distortion appears. This is due to an internal (the plasma boundary is fixed) rotational instability. For $\gamma=20$, $\lambda=11, \delta=0.8, \Omega=0.2, B_{0}=3$ and $f_{n}=10 \%$ the $\mathrm{n}=1$ mode appears when $v_{i \theta} / v_{\text {ther }} \approx 1.4$ and the $\mathrm{n}=2$ mode when $v_{i \theta} / v_{\text {ther }} \approx 1.7$.

\section{Relaxed States for a Flux Core Spheromak Sustained by Helicity Injection}

Helicity injection current drive is generally explained by assuming that the plasma undergoes some form of relaxation that allows for a redistribution of the magnetic flux. Although the minimum energy principle [16] has been successful at explaining some basic features of space and laboratory plasmas, its use in driven systems has been questioned by many authors. In such systems, other principles that allow for the introduction of balance constraints, the use of boundary conditions that include the basic features needed for helicity injection and the inclusion of non uniform/anisotropic resistivity effects could be more appropriate. We employed one such principle, the principle of minimum rate of energy dissipation, to calculate relaxed states of a spheromak sustained by helicity inejction [17]. More specifically, we minimized the Ohmic dissipation rate with the constraints of helicity balance (injection rate=dissipation rate) and $\nabla \cdot \mathbf{B}=\mathbf{0}$. The resulting Euler-Lagrange equation was solved numerically (in 2D) using boundary conditions that include the basic features of the experimental situation.

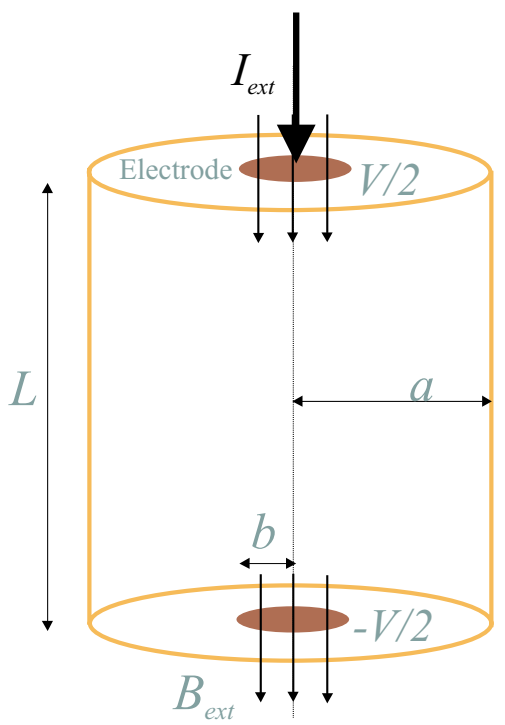

Figure 11. Configuration considered in the calculation of minimum dissipation states.

The configuration considered, shown in Fig. 11, is a simplified version of the one proposed for the PROTOSPHERA experiment [18]. It consists of a flux conserver which is grounded and covered on the inside with a dielectric and two electrodes which are insulated from the flux conserver and kept at voltages of $\pm V_{i n j} / 2$ by a power supply. The top and bottom of the flux conserver are rings of inner radius $b$ and outer radius $a$ and the side is a cylindrical shell of radius $a$ and height $L$. The electrodes are circular, 
with radius $b$, and the entire configuration is axisymmetric $(\partial / \partial \theta \equiv 0)$. We assume that a set of external coils, not shown in the figure, produce a uniform axial magnetic field $\left(B_{\text {ext }}\right)$ on both electrodes. The external field at the electrodes, the voltage and the current flowing through the electrodes are considered external parameters that can be varied independently. The resistivity was considered isotropic but non uniform.

Two types of relaxed states, both with large regions of closed flux surfaces, were found: one has a central core formed by the flux that links the electrodes surrounded by a toroidal region of closed flux surfaces and the other has the open flux wrapped around the closed flux surfaces. Flux contours for both types of solutions are shown in Fig. 12 for $V=600\left(V=V_{i n j} \mu_{0} /\left|B_{\text {ext }}\right| \eta_{0}\right), I=0.72(I=$ $\left.\mu_{0} I_{\text {ext }} / a \pi\left|B_{\text {ext }}\right|\right)$ and uniform resistivity $\left(\eta_{0}=10^{-6} \Omega m\right)$. In Fig. 12a, $\lambda a=3.9228$ while in Fig. 12b, $\lambda a=4.2948$. The solutions shown in Figs. 12a and $12 \mathrm{~b}$ have, respectively, $\lambda\left\langle\lambda_{\text {eigen }}\right.$ and $\lambda>\lambda_{\text {eigen }}$, where $\lambda_{\text {eigen }} a=4.0679$ and corresponds to the solution with $\mathbf{j} . \hat{\mathbf{n}}=0$ everywhere at the boundary. These solutions are relative minima of the dissipation rate and the maximum corresponds to the limit when the injection and dissipation rates go to infinity and $\lambda \rightarrow \lambda_{\text {eigen }}$. The results presented below have $V=600$ and $I=0.72$ and correspond to the case with the open flux on the inside, which is the interesting one for the experiments. a)

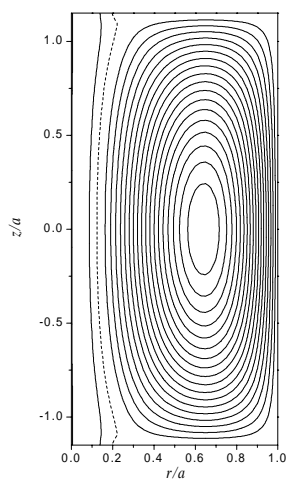

b)

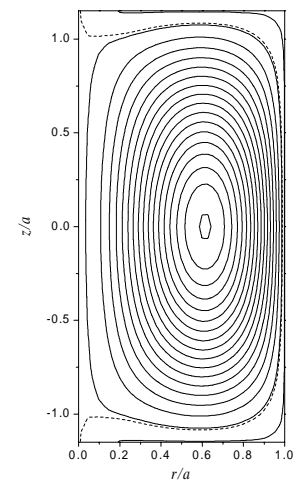

Figure 12. Flux contours for both types of solutions. $\lambda a=3.9228$, (b) $\lambda a=4.2948$.

Since the FCS contains regions of open and closed flux surfaces a resistivity profile that depends on the poloidal flux was employed: $\eta=\eta_{0}\left[1+|\psi| /\left|\psi_{s}\right|\right]^{\alpha}$ in the open flux region and $\eta=\eta_{0}\left[2-1.5\left(|\psi|-\left|\psi_{s}\right|\right) /\left|\psi_{0}\right|\right]^{\alpha}$ in the closed flux region, where $\psi_{s}$ and $\psi_{0}$, are, respectively, the poloidal flux at the separatrix and at the magnetic axis. The parameter $\alpha$ controls the resistivity gradient ( $\alpha=0$ gives uniform resistivity). Fig. 13 shows resistivity profiles at the midplane $(z=0)$ obtained for $\alpha=0,0.5,1$ and 2. As expected, when $\alpha \neq 0$, the resistivity is maximum at the separatrix and minimum at the magnetic axis. Fig. 14 shows radial profiles of $j\left(j=j_{\theta} \mu_{0} a /\left|B_{\text {ext }}\right|\right)$ for the same conditions as in Fig. 13. As $\alpha$ increases, the toroidal current density becomes more peaked and the maximum absolute value shifts outwards.
Finally, Fig. 15. presents plots of the safety factor profile for the same conditions as in Fig. 13. For these calculations the safety factor profile is defined as:

$$
q=\frac{1}{2 \pi} \oint \frac{B}{r B_{p}} d s
$$

where $B_{p}$ is the normalized poloidal field and the integral is calculated following the field line around a single poloidal circuit. When $\alpha=0$, uniform resistivity, $q$ is maximum at the magnetic axis and decreases towards the edge. As $\alpha$ increases, the value at the magnetic axis decreases rapidly while the edge value shows a slight reduction for $\alpha=0.5$ and 1 followed by an increase for $\alpha=2$. This changes in the $q$-profile are clearly related to the changes in the current profile and total current already discussed. For $\alpha=2$, a $50 \%$ increase in the external current (to $I=1.08$ ) increases the value of $q$ at the magnetic axis by $18 \%$ and the value at the edge by $22 \%$.

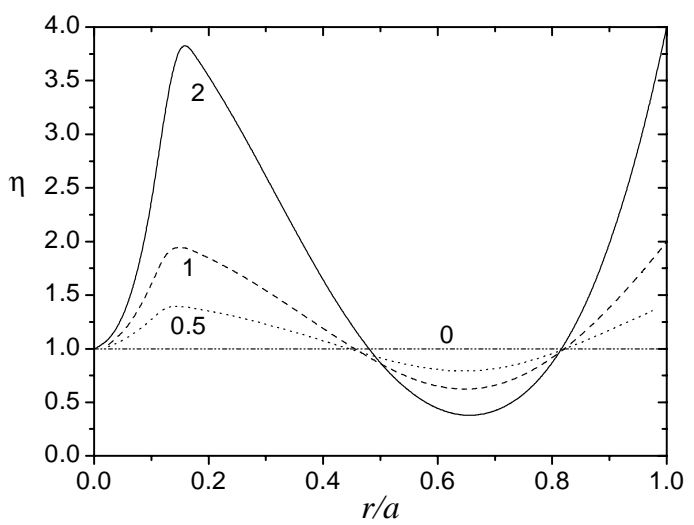

Figure 13. Radial resistivity profiles at $z=0$ for different values of $\alpha$.

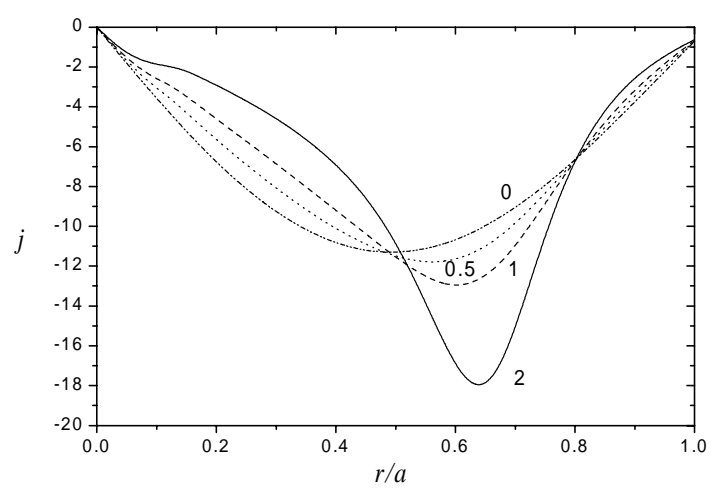

Figure 14. Radial current density profiles at $z=0$ for different values of $\alpha$. 


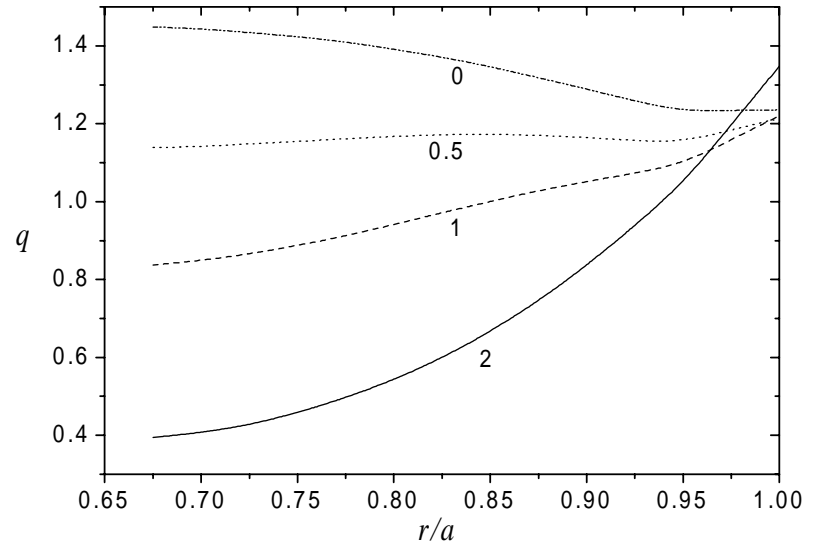

Figure 15. Radial safety factor profiles for different values of $\alpha$.

\section{Summary}

We presented the results of theoretical analysis of three current drive methods which apply to CTs. It was shown that neutral beams can drive a significant current and modify the pressure profiles in high density, $\theta$-pinch produced, FRCs. In lower density, lower field FRCs produced by RMF there are severe limitations to the energy of the neutral particles and the injection geometry. In addition, large reductions in efficiency occur if the amplitude and/or penetration of the $\mathrm{RMF}$ is too large. Neutral beams could provide significant control over the safety factor profiles of Spheromaks but additional studies are needed to determine the effect of fluctuations on beam trapping and the effect of relaxation on the beam current profile.

RMF current drive is very attractive for FRCs but methods to prevent ion spin-up must be developed. It was shown that electron-ion collisions accelerate the ions in the azimuthal direction and large fractions of, fixed, neutral atoms are needed to obtain reasonable current drive efficiencies. In addition, rotational instabilities appear when the azimuthal ion velocity becomes of the order of the thermal velocity. Two dimensional relaxed states of flux core Spheromaks sustained by helicity injection show a large region of closed flux surfaces and significant toroidal current. Changing the resistivity profile modifies the safety factor profile, which can change from one that has a maximum at the magnetic axis (for uniform resistivity) to a tokamak-like $q$ profile. Three dimensional calculations are needed to deter- mine the values of the parameters where a helical relaxed state appears.

\section{Acknowledgements}

This work was partially supported by the Agencia Nacional de Promoción Científica y Tecnológica of Argentina. One of the authors (RAC) would also like to thank financial support from Conselho Nacional de Desenvolvimento Científico e Tecnológico (CNPq, Brazil). The contribution of A.L. Hoffman to the study of NBI in FRCs sustained by $\mathrm{RMF}$ is acknowledged.

\section{References}

[1] M. Tuszewskii, Nuclear Fusion 28, 2033 (1988).

[2] T. R. Jarboe, Plasma Phys. Control. Fusion, 36, 945 (1994).

[3] I. R. Jones, Phys. of Plasmas 6, 1950 (1999).

[4] A. L. Hoffman et al., Nucl. Fusion 43, 1091 (2003).

[5] H. Momota et al., Plasma Physics and Controlled Nuclear Fusion Research, IAEA, Viena, Vol. 3, p. 319 (1993).

[6] T. Asai et al., Phys. Plasmas 7, 2294 (2000).

[7] E. B. Hooper, L. D. Pearlstein, and R. H. Bulmer, Nucl. Fusion 39, 863 (1999).

[8] T. R. Jarboe, Fusion Technol., 36, 85 (1999).

[9] A.F. Lifschitz R. Farengo, and N. R. Arista, Nucl. Fusion 42 (2002) 863 .

[10] A. L. Hoffman, Nucl. Fusion 40, 1523 (2000).

[11] A. F. Lifschitz, R. Farengo, and N. R. Arista, Plasma Phys. Control. Fusion 44, 1979 (2002).

[12] R. D. Milroy, Phys. Plasmas, 6, 2771 (1999).

[13] R. D. Milroy, Phys. Plasmas, 7, 4135 (2000).

[14] W. N. Hugrass and R. C. Grim, J. Plasma Phys. 26, 455 (1981).

[15] W. N. Hugrass J. Plasma Phys. 28, 369 (1982).

[16] J. B. Taylor, Phys. Rev. Lett. 33, 1139 (1974).

[17] R. Farengo and K. I. Caputi, Plasma Phys. and Contr. Fusion 44, 1707 (2002).

[18] F. Alladio, Private communication 2000. The complete PROTO-SPHERA proposal can be downloaded from: http://www.frascati.enea.it/ProtoSphera 